\title{
Differential Linear Logic and Processes
}

\author{
Thomas Ehrhard \\ Preuves, Programmes \& Systèmes \\ Université Paris Diderot and CNRS
}

In Linear Logic, the tensor/par and plus/with dualities are lost when exponentials come in.

- The "?" modality is introduced by the weakening and dereliction rules, and contraction allow to contract two occurrences of a formula ? A (from an unique premise sequent) into a single one

- whereas the "!" modality can be introduced only by mean of a promotion rule.

By adding new rules for the "!" modality, one retrieves, in the exponential fragment, a duality and a symmetry similar to that of the multiplicative fragment. These new rules are

- coweakening and cocontraction which are new ways of introducing "!" formulas

- and cocontaction, which allow to contract two occurrences of ! $A$ (from two different premise sequents) into a single one.

Corresponding reduction (cut-elimination) rules are added, which express operationally this new !/? symmetry. These reduction rules are semantically justified, when interpreting the new logical rules for "!" as standard operations on functions (in particular, codereliction corresponds to differentiation of a function at point 0 of a vector space). This extended linear logic is called Differential Linear Logic (DLL).

This new symmetry adds expressive power to linear logic. In particular, we show how a fragment of the $\pi$-calculus can be translated into differential interaction nets (a system if interaction nets where cells correspond to rules of DLL) and how the dereliction/codereliction reductions of this differential interaction net simulate the reductions of the process. Last, we present a simple denotational model of differential interaction nets, in a category of sets and relations. This model, which is also a natural model of the pure lambda-calculus ( $\beta$ and $\eta$ ), becomes therefore a model of the considered fragment of the $\pi$-calculus, and we explore some of its properties.

Please use the following format when citing this chapter:

Ehrhard, T., 2008, in IFIP International Federation for Information Processing, Volume 273; Fifth IFIP International Conference on Theoretical Computer Science; Giorgio Ausiello, Juhani Karhumäki, Giancarlo Mauri, Luke Ong; (Boston: Springer), pp. 283. 